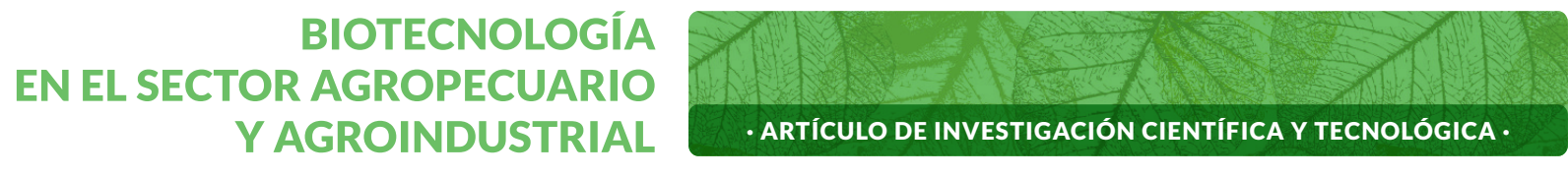

\title{
Antagonismo in vitro por consorcios de Trichoderma sp. y Aspergillus sp. contra el fitopatógeno Sclerotinia sp*
}

\author{
In vitro antagonism by consortium of \\ Trichoderma sp. and Aspergillus sp. against \\ the phytopathogen Sclerotinia sp
}

\section{Antagonismo in vitro por Trichoderma sp. e Aspergillus sp. contra o fitopatógeno Sclerotinia sp}

ZUBIETA-CORONADO, DIEGO-ARTURO ${ }^{1}$; ECHEVERRY-PRIETO, LENA-CAROLINA²; ZAFRA-MEJÍA, CARLOS-ALFONSO ${ }^{3}$

Historial del Artículo

Recibido para evaluación: 19 de Agosto 2019.

Aprobado para publicación: 13 de Octubre 2020.

* Título del proyecto de origen: "Evaluación in vitro e in vivo del control biológico hecho por Trichoderma sp. y Aspergillus sp. sobre el fitopatógeno Sclerotinia sp. en lechuga (Lactuca sativa) variedad Batavia". Financiación: Centro de Investigaciones y Desarrollo Científico (CIDC) de la Universidad Distrital Francisco José de Caldas. Bogotá, Colombia. Culminación: 27 de mayo 05 de 2019.

1 Universidad Distrital Francisco José de Caldas, Facultad del Medio Ambiente y Recursos Naturales, Grupo de investigación GIIA-UD. Especialización en Higiene y Salud Ocupacional. Bogotá, Colombia. https://orcid.org/0000-0003-3568-8282

2 Universidad Distrital Francisco José de Caldas, Facultad del Medio Ambiente y Recursos Naturales, Grupo de investigación GIIA-UD. Maestría en Ciencias Biológicas. Bogotá, Colombia. https://orcid.org/0000-0002-6940-4383

3 Universidad Distrital Francisco José de Caldas, Facultad del Medio Ambiente y Recursos Naturales, Grupo de investigación GIIA-UD. Doctorado en Ingeniería Ambiental. Bogotá, Colombia. https://orcid.org/0000-0002-4061-4897 


\section{RESUMEN}

Los hongos como controladores biológicos son importantes en el desarrollo de bioproductos para una agricultura sostenible en el manejo de fitopatógenos como Sclerotinia sp., causante de la podredumbre blanca en cultivos de lechuga, puesto que la viabilidad infecciosa en el suelo dura hasta cinco años. El cultivo de lechuga es esencial en la economía agrícola de Colombia, y Sclerotinia sp. causa pérdidas hasta del $50 \%$ de los cultivos. El objetivo de este trabajo fue evaluar In vitro la capacidad antagónica de tres cepas de Trichoderma sp. Tr1, Tr2 y Tr3 y tres de Aspergillus sp. As1, As2 y As3 contra Sclerotinia sp Scl., individualmente y en consorcios. La recuperación, mantenimiento y evaluación de los hongos se realizó mediante los medios PDA, Jugo V8 y Miel-Avena. Las cepas controladoras - $\operatorname{Tr} 1, \operatorname{Tr} 2, \operatorname{Tr} 3$, As1, As2 y As3- se enfrentaron entre ellas y en consorcios contra el fitopatógeno con técnica dual. Los consorcios As1+As2+Tr2, As1+As2+Tr1, y As1+As2+Tr3 tuvieron un grado de inhibición sobre el fitopatógeno, siendo más eficiente la combinación As1+As2+Tr1 -85,1\%-; e individualmente las cepas Tr2 y As1 con una eficiencia del 95,6\% y 95,1\%, respectivamente. Se corroboró que el crecimiento de Scl. empleando los consorcios no fue mayor al 14,9\% en el mejor tratamiento. En ninguno de los enfrentamientos con los consorcios se evidenció la formación de esclerocios, la estructura de resistencia en el proceso de infección y persistencia en suelos a los diez días de evaluación.

\section{ABSTRACT}

Fungi as biological controllers are important in development of bioproducts for sustainable agriculture in the management of phytopathogens such as Sclerotinia sp., which causes white rot in lettuce, crops insomuch as infectious viability in soil lasts up until five years. Lettuce cultivation is essential in Colombia's agricultural economy, and Sclerotinia sp. causes losses until 50\% of crops. The aim of this work was to evaluate in vitro the antagonistic capacity of three strains of Trichoderma sp. Tr1, Tr2 and Tr3 and, three strains of Aspergillus sp. As1, As2 and As3 against Sclerotinia sp Scl., individually and in consortia. Recovering, maintenance and evaluation of fungi was carried out by mediums as PDA, V8 Juice and Honey-Oat. The control strains -Tr1, Tr2, Tr3, As1, As2 and As3- were confronted among them and in consortia against the phytopathogen with dual technique. The consortia As1+As2+Tr2, As1+As2+Tr1, and As $1+A s 2+\operatorname{Tr} 3$ had a degree of inhibition on the phytopathogen, being more efficient the combination As1+As2+Tr1 -85,1\%-; and individually the strains Tr2 and As1 with an efficiency of 95,6\% and 95,1\%, respectively. It was corroborated that the growth of Scl. using the consortia was not more than $14,9 \%$ in the best treatment. In none of the confrontations with the consortia was evidence of sclerotia formation, resistance structure for infection process and persistence in soils after to ten days of evaluation.

Cómo citar este artículo: ZUBIETA-CORONADO, DIEGO-ARTURO; ECHEVERRYPRIETO, LENA-CAROLINA; ZAFRA-MEJÍA, CARLOS-ALFONSO. Antagonismo in vitro por consorcios de Trichoderma sp. y Aspergillus sp. contra el fitopatógeno Sclerotinia sp. Biotecnología en el sector agropecuario y agroindustrial, v. 19, n. 1, 2021, p. 16-31. Doi: https://doi.org/10.18684/BSAA(19)16-31

\section{PALABRAS CLAVE:}

Antagonistas fúngicos; Consorcio; Control biológico; Podredumbre blanca; Fitopatógeno; Trichoderma sp; Aspergillus sp; Sclerotinia sp.

\section{KEYWORDS:}

Fungal antagonists; Consortium; Biological control; White rot, Phytopathogen; Trichoderma sp; Aspergillus sp; Sclerotinia sp.

\section{PALAVRAS-CHAVE:}

Antagonistas de fungos; Consórcio; Controle biológico; Podridão branca; Fitopatógenos; Trichoderma sp; Aspergillus sp; Sclerotinia sp. 


\section{RESUMO}

Os fungos, como controladores biológicos, são importantes no desenvolvimento de bioprodutos para a agricultura sustentável no manejo de fitopatógenos como Sclerotinia sp., que causa podridão branca nas culturas de alface, visto que a viabilidade infecciosa no solo dura até cinco anos. O cultivo da alface é essencial na economia agrícola da Colômbia e Sclerotinia sp. causa perdas até $50 \%$ das lavouras. O objetivo deste trabalho foi avaliar in vitro a capacidade antagônica de três linhagens de Trichoderma sp. Tr1, Tr2 e Tr3 e, três linhagens de Aspergillus sp. As1, As 2 e As3 contra Sclerotinia sp Scl., individualmente e em consórcios. A recuperação, manutenção e avaliação de fungos foram realizadas por meios como PDA, Suco V8 e Aveia-Mel. As cepas de controle - Tr1, Tr2, Tr3, As1, As2 e As3 - foram confrontadas entre si e em consórcios contra o fitopatógeno com técnica dupla. Os consórcios As1 + As2 + Tr2, As1 + As2 + Tr1 e As1 + As2 + Tr3 apresentaram um grau de inibição no fitopatógeno, sendo mais eficiente a combinação As1 + As2 + Tr1 -85,1\% -; e individualmente as linhagens Tr2 e As1 com uma eficiência de 95,6\% e 95,1\%, respectivamente. Foi corroborado que o crescimento de Scl. o uso dos consórcios não foi superior a 14,9\% no melhor tratamento. Em nenhum dos confrontos com os consórcios houve evidência de formação de escleródios, estrutura de resistência ao processo de infecção e persistência no solo até dez dias de avaliação.

\section{INTRODUCCIÓN}

La lechuga (Lactuca sativa) es originaria del Mediterráneo oriental y del occidente de Asia [1, 2], y su cultivo puede realizarse a nivel del mar hasta 2600 msnm [3]. Es un vegetal rico en sustancias orgánicas, vitaminas, minerales y metabolitos secundarios como los terpenoides, flavonoides y fenoles, que aportan a su particular sabor y alto valor nutritivo [2, 4]. En Colombia, este cultivo se desarrolla principalmente en Cundinamarca, Antioquia, Nariño y Norte de Santander. En Cundinamarca es uno de los cultivos pilares de la economía rural, y durante el año 2018 se produjeron 23.525 ton. Durante el mismo año, a nivel nacional la producción de lechuga fue de 49982,58 ton y el rendimiento del cultivo en 2018 fue de 238 ton/ha y en 2017 de 233,63 [5, 6].

De acuerdo con Pérez et al. [7], las perdidas en los cultivos de lechuga en la sabana de Bogotá por infección por Sclerotinia sp están entre el 30-50\% de la producción total. Este hongo puede infectar a más de 400 especies de plantas, incluidos cultivos y hortalizas económicamente importantes [8]. El fitopatógeno Sclerotinia sp. se puede propagar por ascosporas, lo que favorece su diseminación a plantas adyacentes y forma esclerocios como estructuras de resistencia. El inóculo puede infectar tallos, hojas, flores y raíces. Las plantas pueden ser infectadas desde su etapa de semilla hasta su madurez, y su incidencia está relacionada con la cantidad de esclerocios que se encuentran en el suelo. Adicionalmente, Sclerotinia sp. puede persistir en el suelo durante varios años o en lotes de semillas y, cuando existen las condiciones ambientales adecuadas, germina dando lugar a hifas infecciosas o produce un apotecio que libera ascoesporas producidas sexualmente [7-9]. La viabilidad de los esclerocios puede alcanzar veinte años en latencia y su dispersión es rápida con los procesos de arado. La supervivencia de los esclerocios en el suelo dificulta el manejo de Sclerotinia sp., por la supervivencia aún en condiciones adversas para el fitopatógeno [10,11].

El manejo tradicional de las plagas se hace con agentes químicos y se estima que en Colombia se emplean unos 600 plaguicidas, siendo una fuente de contaminación en suelos y aguas, los cuales son responsables de afecciones a los seres vivos de dichos ecosistemas y sobre la salud humana [12, 13]. El uso excesivo de agroquímicos puede incrementar la resistencia a estos compuestos de los hongos fitopatógenos, haciendo cada vez más difícil su manejo. Por consiguiente, el control biológico es una herramienta alternativa amigable con el ambiente para el manejo de plagas y enfermedades, que se basa en el empleo de organismos biológicos y sus productos metabólicos con el fin de reducirla, eliminarla o controlarla [14, 15]. Según la literatura, se han realizado varios trabajos para evaluar organismos antagonistas para el control de Sclerotinia sp., como por ejemplo: Trichoderma sp., Aspergillus flavipes, Muscodor yucatanensis, Penicillium commune, Bacillus sp., Clonostachys sp., Paecilomyces lilacinus, Pseudomonas sp. y Coniothyrium sp. [9, 15-21]. 
Recientemente, el uso de hongos endófitos como antagonistas es una opción llamativa para el manejo integrado de plagas por su mínimo impacto sobre el ambiente [15]; por tal motivo, en este trabajo de investigación se aislaron e identificaron de suelos rizosféricos tres cepas de Trichoderma sp. y tres cepas de Aspergillus sp. autóctonos, para evaluar la relación antagónica de éstos hongos individualmente y mediante consorcios frente al fitopatógeno Sclerotinia sp. Este fitopatógeno fue aislado directamente de lechugas que presentaban los síntomas de la podredumbre blanca. Por último, se estudiaron agrupaciones de hongos antagonistas para poder inhibir el desarrollo de este fitopatógeno en cultivos de lechuga.

\section{MÉTODO}

\section{Sitio de muestreo}

El sitio seleccionado para la recolección de muestras de suelo fue en la ciudad de Bogotá, Colombia. Las muestras se recolectaron de tres zonas con las siguientes coordenadas: Zona 1: $4^{\circ} 35^{\prime} 49,55^{\prime \prime} \mathrm{N}-7^{\circ} 03^{\prime} 51,86^{\prime \prime} \mathrm{W}$, Zona 2: 4³5'51,03" N - 7403'51,57' W y Zona 3: 4³5'50,80" N - 7403'51,64" W y se rotularon como MT01, MT02 y MT03, respectivamente. La ubicación hace parte de los cerros orientales de Bogotá, con una humedad del $81 \%$, una altura aproximada de $2675 \mathrm{msnm}$ y una temperatura que oscila durante el día entre los 6 y $18^{\circ} \mathrm{C}$.

\section{Recuperación y aislamiento de hongos con potencial biocontrolador}

El muestreo de suelos rizosféricos se realizó aleatoriamente en una sección de $20 \times 20 \mathrm{~cm}$ hasta obtener $10 \mathrm{~g}$ por cada zona ( $n=3)$, de acuerdo con la metodología reportada por Cline [22]. Las muestras de suelos recolectadas se sembraron en los medios de cultivo PDA, Jugo V8 y Miel-Avena usando el método de cuadricula. Se incubaron por un lapso de 10 días a una temperatura de $25^{\circ} \mathrm{C}$ para que los hongos desarrollaran sus estructuras (i.e., micelio, conidias, fiálides, conidióforos, y vesículas), lo cual permitió la identificación macroscópica y microscópica de cada aislamiento [23-27]. Se recuperaron seis hongos en total y posterior a la identificación se etiquetaron los aislamientos como: Tr1, Tr2 y Tr3 para Trichoderma sp. y As1, As2 y As3 para Aspergillus sp.

\section{Recolección de material vegetal, recuperación y mantenimiento del hongo fitopatógeno}

Se recolectaron lechugas que provenían del municipio de Mosquera (Cundinamarca, Colombia) y que presentaban las características de infección producidas por el hongo Sclerotinia sp., como marchitamiento de hojas externas, lesiones de color marrón en el tejido foliar, pudrición blanda acuosa, crecimiento de micelio de color blanco y/o presencia de esclerocios [28]. Estas muestras fueron recolectadas del centro de acopio Corporación de Abastos de Bogotá S.A. (CORABASTOS). Las muestras se conservaron a $4^{\circ} \mathrm{C}$ y se transportaron al laboratorio de Microbiología y bioprospección medioambiental (MiBioMA) de la Universidad Distrital Francisco José de Caldas (Bogotá, Colombia). El aislamiento de Sclerotinia sp. se realizó mediante la técnica expuesta por Sánchez [29]. Los cortes de 5-10 mm de las hojas de lechuga infectadas se colocaron en una solución de cloro al 10\% por 20 segundos, se lavaron tres veces con agua destilada, se colocaron en papel secante, y se ubicaron en el centro de los medios de cultivo PDA, Jugo V8 y Miel-Avena. Éstas se incubaron por diez días a una temperatura de $25^{\circ} \mathrm{C}$.

Adicionalmente, se realizó un aislamiento a partir de esclerocios, desinfectándolos con una mezcla de hipoclorito al $70 \%$, fenol al $5 \%$ y alcohol etílico por un tiempo de 5 minutos; los esclerocios se lavaron con agua destilada estéril y con una cuchilla estéril se cortaron para sembrarlos en los medios de cultivo de evaluación. La temperatura y tiempo de incubación fue de $25^{\circ} \mathrm{C}$ por 10 días [29]. Se identificaron macroscópica y microscópica del hongo fitopatógeno se realizó de acuerdo con Saharan, G.S. \& Mehta, N [30]. 


\section{Pruebas de antagonismo}

Antes de realizar las pruebas de antagonismo se estimó el crecimiento radial de cada hongo aislado en los tres medios de cultivo de evaluación, midiendo el diámetro de las colonias los días 3, 7 y 10. La esporulación de Trichoderma sp y Aspergillus se realizó según Pedroza et al. [31] al culminar los 10 días de incubación. Para Sclerotinia se contaron los esclerocios a los 10 días de crecimiento.

Se realizaron pruebas de antagonismo contra el fitopatógeno a través de la técnica dual [32], con las tres cepas de Trichoderma sp. (Tr1, Tr2 y Tr3) y las tres cepas de Aspergillus sp. (As1, As2 y As3). También, se evalúo el antagonismo entre ellos, así: As1 vs. Tr1, As1 vs. Tr2, As1 vs Tr3, As2 vs. Tr1, As2 vs. Tr2, As2 vs Tr3, As3 vs. Tr1, As3 vs. Tr2, As3 vs Tr3. En cada caja de Petri se marcó una línea para dividir en dos partes iguales el área de siembra, y en la mitad de cada lado se dispusieron discos de medio de cultivo con micelio de los hongos a enfrentar de $5 \mathrm{~mm}$ de diámetro [33]. Los quince antagonismos se incubaron por diez días a $25^{\circ} \mathrm{C}$. Cada enfrentamiento se realizó en los medios PDA, Jugo V8 y Miel-Avena. La evaluación se realizó de acuerdo con Bell et al. (1982), referenciado por Corrêa et al. [34]; y se modificó cuando se enfrentaron los posibles biocontroladores teniendo la escala de notas No. 1 (Cuadro 1 y Figura 1), con base en el método de similaridad de Jaccard [35].

Posteriormente, se evaluó el efecto antagónico entre consorcios de los posibles hongos biocontroladores contra Sclerotinia sp., así: Scl vs. Tr1+As1+As2, Scl vs. Tr1+As1+As2, y Scl vs. Tr3+As2+As3. Todas las pruebas de antagonismo se realizaron en los tres medios de cultivo y se incubaron por diez días a $25^{\circ} \mathrm{C}$. Como control del crecimiento para cada hongo se sembró en los tres medios mencionados por punción central individualmente, y se comparó el crecimiento con lo observado en cada enfrentamiento. Para evaluar el efecto antagonista entre cada hongo y los consorcios frente al fitopatógeno, se definió una segunda escala de notas (Cuadro 2 y Figura 1) de acuerdo con el método de similaridad de Jaccard [35].

Cuadro 1. Escala de notas para valorar el antagonismo entre Trichoderma sp. y Sclerotinia sp.

\begin{tabular}{|c|l|c|}
\hline Clase & \multicolumn{1}{|c|}{ Característica } & $\begin{array}{c}\text { Valoración } \\
\text { binaria }\end{array}$ \\
\hline 1 & Sobrecrecimiento de Trichoderma: colonizó toda la superficie del medio y redujo la colonia de Aspergillus. & 1 \\
\hline 2 & Sobrecrecimiento de Trichoderma: colonizó al menos 2/3 de la superficie del medio. & 1 \\
\hline 3 & Sobrecrecimiento de Aspergillus: colonizó toda la superficie del medio y redujo la colonia de Trichoderma. & 0 \\
\hline 4 & Sobrecrecimiento de Aspergillus: colonizó al menos 2/3 de la superficie del medio. & 0 \\
\hline 5 & Trichoderma y Aspergillus: colonizaron medio a medio (más que 1/3 y menor que $2 / 3)$. Uno no se sobrepuso al otro. & 0 \\
\hline
\end{tabular}

Cuadro 2. Escala de notas para valorar el antagonismo entre los consorcios y Sclerotinia sp.

\begin{tabular}{|c|l|c|}
\hline Clase & \multicolumn{1}{|c|}{ Característica } & $\begin{array}{c}\text { Valoración } \\
\text { binaria }\end{array}$ \\
\hline 1 & $\begin{array}{l}\text { Sobrecrecimiento del antagonista o consorcio, que colonizó toda la superficie del medio y redujo la colonia } \\
\text { del patógeno. }\end{array}$ & 1 \\
\hline 2 & Sobrecrecimiento del antagonista o consorcio, que colonizó al menos 2/3 de la superficie del medio. & 1 \\
\hline 3 & $\begin{array}{l}\text { El antagonista o consorcio y el patógeno colonizaron medio a medio (más que 1/3 y menor que 2/3). Uno no } \\
\text { se sobrepuso al otro. }\end{array}$ & 0 \\
\hline 4 & $\begin{array}{l}\text { El hongo patógeno colonizó al menos 2/3 de la superficie del medio y resistió la invasión por el antagonista } \\
\text { o consorcio. }\end{array}$ & 0 \\
\hline 5 & Sobrecrecimiento del hongo patógeno que colonizó toda la superficie del medio. & 0 \\
\hline
\end{tabular}


Figura 1. Distribución de crecimiento en cajas de Petri de acuerdo con las escalas de notas 1 y 2.

Crecimiento de enfrentamiento - Escala de notas 1.

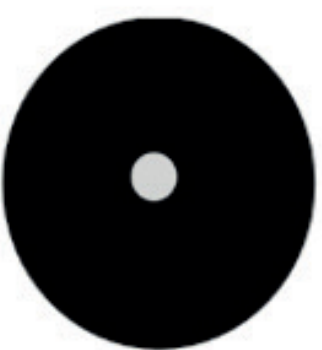

Clase 1

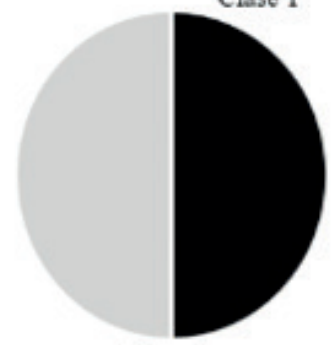

Clase 3

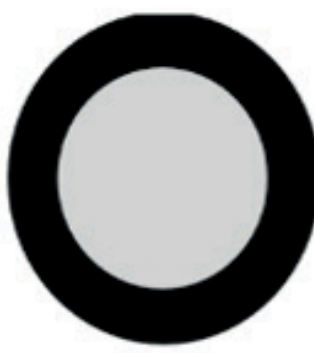

Clase 4

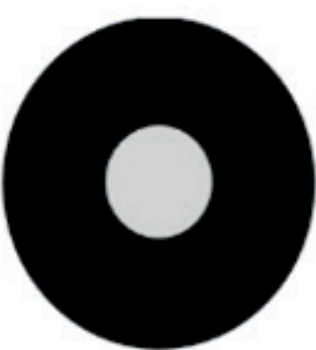

Clase 2

Colonia de Aspergillus sp.

Colonia de Trichoderma sp.

Crecimiento de enfrentamiento - Escala de notas 2.

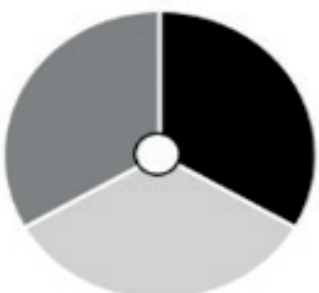

Clase 1

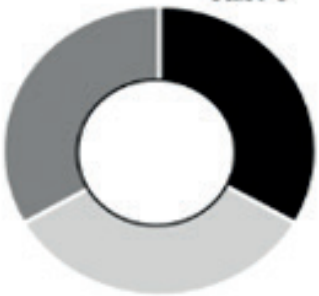

Clase 3

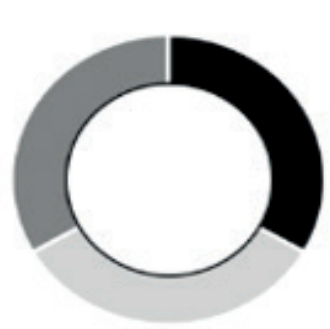

Clase 4

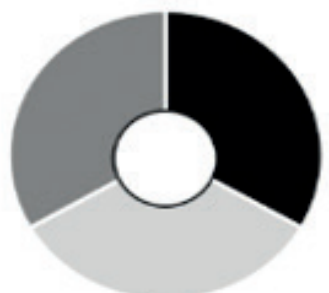

Clase 2

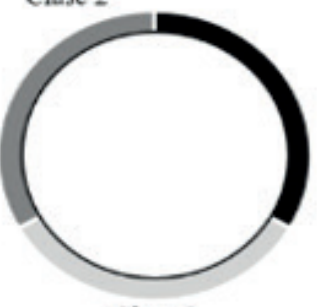

Clase 5

Colonia 1 de Aspergillus sp.

Colonia 2 de Aspergillus sp.

Colonia de Trichoderma sp.

Colonia de Sclerorinia sp. 


\section{Diseño experimental y análisis estadístico}

Se aplicó un diseño experimental basado en la clasificación de conglomerados jerárquicos con medida de similaridad de Jaccard [28], donde se distinguieron las relaciones entre cada una de las cepas Tr1, Tr2, Tr3, As1, As2, As3 y Scl. En la escala de similaridad se obtuvieron datos binarios, en donde cero hizo referencia a que no hubo un antagonismo mayor al 50\%. En la escala de Notas 1, son las clases 2, 4 y 5; y para la escala de notas 2, son las clases 3, 4 y 5 . La valoración uno (1) fue para el antagonismo igual o superior al 50\%, lo que correspondió en la escala de notas 1 a las clases 1 y 3 ; para la escala de notas 2 , las clases 1 y 2 (Figura 1).

Por último, la distribución normal de los datos se verificó mediante una prueba de Shapiro-Wilk ( $p$-valor > 0,05) y el estadístico de Levene ( $p$-valor $<0,05$ ). Se determinó también el porcentaje de relación, y se comprobó el grado de similaridad mediante el método de Jaccard. Se utilizó un análisis de varianza de una vía y la prueba Tukey para estudiar las diferencias entre las variables consideradas. Todos los análisis fueron realizados mediante el programa informático SPSS V.19.

\section{RESULTADOS}

\section{Cepas fúngicas}

Los hongos Trichoderma sp. y Aspergillus sp. encontrados en los suelos rizosféricos son cosmopolitas ampliamente conocidos [36, 37]. En particular, el hongo Trichoderma sp., que desde hace décadas se conoce por sus propiedades como biocontrolador y capacidad de generar lisis en las paredes celulares de los hongos fitopatógenos, como en los esclerocios por las enzimas quitinasas, proteasas y glucanasas. Igualmente, secretan diversos metabolitos con acción antibiótica, que suministran tolerancia a condiciones estresantes y favorecen la competencia por espacio y sustrato [15, 17, 33]. En relación con Aspergillus sp., son pocos los estudios que han evaluado este hongo como biocontrolador [36, 37], siendo un campo que debe seguir profundizándose. La mayoría de las investigaciones se han enfocado en las especies fitopatógenas, contaminantes de alimentos, generadoras de enfermedades en humanos y animales; como también en especies con aplicación biotecnológica para la producción de enzimas como las xilanasas, amilasas, pectinasas o de metabolitos como ácidos orgánicos y proteínas homologas y/o heterólogas [38, 39].

De las tres zonas de muestreo se obtuvieron e identificaron seis aislados de hongos, tres del género Trichoderma sp. y las restantes al género Aspergillus sp. Dichos aislamientos se incorporaron a la colección de hongos del laboratorio de microbiología y bioprospección medioambiental -MiBioMA- de la Universidad Distrital Francisco José de Caldas y se identificaron como: $\operatorname{Tr} 1, \operatorname{Tr} 2$ y $\operatorname{Tr} 3$ para las cepas de Trichoderma sp.; y As1, As 2 y As3, para las cepas de Aspergillus sp. La obtención del hongo Sclerotinia sp., Scl, fue exitoso a partir de esclerocios posterior al proceso de desinfección como fue descrito.

Luego del proceso de aislamiento de los hongos, se determinö el crecimiento de cada uno en los medios de evaluación (Cuadro 3) y en los 10 días de incubación el promedio de crecimiento fue para As1: $8 \mathrm{~cm}$, As2: 7,75 $\mathrm{cm}$, AS3: 7,1 cm, $\operatorname{Tr} 1: 7,9 \mathrm{~cm}$, Tr2: 8,05 cm y Tr3: 8,05 cm. Con respecto a la esporulación, en los 10 días de crecimiento se obtuvo un promedio de conidios/mL para As 1: 9,30E+06, AS2: 1,95E+06, As3: 9,23E+05, $\operatorname{Tr} 1$ : 1,35E+07, Tr2: 3,33E-07 y Tr3: 2,14E-07. Para el caso de Scl, se determinó el número de esclerocios y se obtuvo un promedio de 11 esclerocios/10 días. 
Cuadro 3. Crecimientos miceliales en los días 3, 7 y 10 de los asilamientos, esporulación y esclerocios en 10 días de evaluación.

\begin{tabular}{|c|c|c|c|c|c|}
\hline HONGO & MEDIO & $\begin{array}{c}\text { Esporulación } \\
\text { (conidios/mL) Día } 10\end{array}$ & $\begin{array}{c}\text { Crecimiento día } \\
3(\mathrm{~cm})\end{array}$ & $\begin{array}{c}\text { Crecimiento día } \\
7(\mathrm{~cm})\end{array}$ & $\begin{array}{c}\text { Crecimiento día } \\
10(\mathrm{~cm})\end{array}$ \\
\hline \multirow{3}{*}{ As1 } & PDA & $1,90 E+06$ & 2,1 & 5,0 & 7,1 \\
\hline & AVENA & $2,10 E+06$ & 2,4 & 5,7 & 8,1 \\
\hline & V8 & $2,50 E+06$ & 2,3 & 5,5 & 7,8 \\
\hline \multirow{3}{*}{ As2 } & PDA & $1,71 \mathrm{E}+06$ & 2,1 & 4,9 & 7 \\
\hline & AVENA & $1,98 \mathrm{E}+06$ & 2,4 & 5,5 & 7,9 \\
\hline & V8 & $1,91 \mathrm{E}+06$ & 2,3 & 5,3 & 7,6 \\
\hline \multirow{3}{*}{ As3 } & PDA & $8,70 E+05$ & 2,1 & 4,9 & 7 \\
\hline & AVENA & $9,40 E+05$ & 2,1 & 5,0 & 7,1 \\
\hline & V8 & $9,10 E+05$ & 2,1 & 4,9 & 7 \\
\hline \multirow{3}{*}{ Tr1 } & PDA & $1,09 \mathrm{E}+07$ & 2,2 & 5,1 & 7,3 \\
\hline & AVENA & $1,23 E+07$ & 2,5 & 5,7 & 8,2 \\
\hline & V8 & $1,43 E+07$ & 2,3 & 5,4 & 7,7 \\
\hline \multirow{3}{*}{$\operatorname{Tr} 2$} & PDA & $2,91 \mathrm{E}+07$ & 2,2 & 5,1 & 7,3 \\
\hline & AVENA & $3,18 \mathrm{E}+07$ & 2,4 & 5,7 & 8,1 \\
\hline & V8 & $3,34 \mathrm{E}+07$ & 2,3 & 5,5 & 7,8 \\
\hline \multirow{3}{*}{$\operatorname{Tr} 3$} & PDA & $1,80 \mathrm{E}+07$ & 2,2 & 5,1 & 7,3 \\
\hline & AVENA & $2,21 \mathrm{E}+07$ & 2,4 & 5,7 & 8,1 \\
\hline & V8 & $2,11 E+07$ & 2,3 & 5,5 & 7,8 \\
\hline HONGO & MEDIO & Esclerocios Día 10 & $\begin{array}{c}\text { Crecimiento día } \\
3(\mathrm{~cm})\end{array}$ & $\begin{array}{c}\text { Crecimiento día } \\
7(\mathrm{~cm})\end{array}$ & $\begin{array}{c}\text { Crecimiento día } \\
10(\mathrm{~cm})\end{array}$ \\
\hline \multirow{3}{*}{$\mathrm{Scl}$} & PDA & 9 & 2,4 & 5.9 & 8,1 \\
\hline & AVENA & 11 & 2,7 & 6.2 & 9 \\
\hline & V8 & 10 & 2,5 & 5.8 & 8,6 \\
\hline
\end{tabular}

Las cepas de Trichoderma sp. se distinguieron macroscópicamente por no formar borde y por tener un crecimiento difuso en la superficie, similar a un "césped". Se observó un micelio de color amarillo hasta tonalidades verdes, que con el tiempo se volvió terroso. Microscópicamente, las fiálides fueron cortas y ahusadas, como una especie de botella, los conidióforos estaban apartados en ángulo obtuso en relación con las hifas. Los conidios se observaron con forma elíptica a globosa y en forma de racimos, similares a pequeños arboles ramificados.

Las colonias de los aislamientos de Aspergillus sp. tuvieron una coloración amarillo oscuro verdoso o marrón y algodonosas; la esporulación fue profusa y las colonias se tornaban granulosas. Microscópicamente, las vesículas fueron pequeñas en forma de cúpula, con métulas sostenidas, fiálides en hilera, y cadenas con conidias elípticas y aleuriosporas unidas a hifas sumergidas.

Trichoderma sp. y Aspergillus sp. producen enzimas extracelulares capaces de degradar grandes polímeros como la celulosa, la pectina, la lignina y el almidón [37, 38], lo que explica que el mayor crecimiento y esporulación en todos los asilamientos haya sido en el medio de cultivo Miel-Avena, seguido por el medio Jugo V8 y, por último, por el medio PDA. De acuerdo con Di Cologna et al. [40], la relación de enzimas excretadas al medio por los hongos Trichoderma sp. y Aspergillus sp. varían de acuerdo con las condiciones ambientales y nutricionales respondiendo, con un conjunto de enzimas diferentes en cada situación. De esta manera, la relación de sustratos complejos es proporcional a la producción de enzimas, pero cuando la fuente de carbono es sencilla la producción de enzimas se reprime. 


\section{Pruebas de Antagonismo}

En el primer enfrentamiento dual se evaluó el efecto antagónico de las cepas de Aspergillus sp. As1, As2 y As3 frente a las cepas de Trichoderma sp. y se valoró según la escala de notas No. 1 (Cuadro 1). En el Cuadro 4 están las clases luego de valorar el efecto antagónico de cada enfrentamiento allí descrito. Se encontró que el 48,2\% de los 27 enfrentamientos correspondieron a un crecimiento masivo de una cepa de Trichoderma sp., colonizando la colonia del Aspergillus sp. Un 7,41\% correspondió al crecimiento masivo de una cepa de Aspergillus sp. sobre Trichoderma sp. En el 29,6\% de los enfrentamientos Trichoderma sp. creció en 2/3 de la caja de Petri, y en un $11,1 \%$ lo hicieron las cepas de Aspergillus sp. Solamente en un 3,70\% de los enfrentamientos las colonias crecieron ocupado prácticamente el mismo espacio sin sobreponerse las colonias (Clase 5, cuadro 1).

Igualmente, se pudo sugerir que la composición de los medios de cultivo influyó en el comportamiento de los promisorios antagonistas de Sclerotinia sp. cuando se evalúan conjuntamente y los antagonismos no fueron homogéneos en los 27 enfrentamientos duales. Es decir, en el medio de cultivo PDA la cepa As1 frente a las cepas $\operatorname{Tr} 1$ y $\operatorname{Tr} 3$ colonizaron aproximadamente $2 / 3$ de la caja; con la cepa Tr2 fue diferente el comportamiento, creciendo en la totalidad de la caja y sobre la colonia As1. En cuanto a la cepa As2, esta creció hasta 1/3 en el medio de cultivo de los $\operatorname{Tr} 1$ y $\operatorname{Tr} 2$; y en el enfrentamiento con la cepa $\operatorname{Tr} 3$, tanto Aspergillus sp. como Trichoderma sp. crecieron cada uno hasta $1 / 3$ de la caja y las colonias se mantuvieron separadas. Para la cepa As 3 , en los tres enfrentamientos contra cualquier cepa de Trichoderma sp., la colonia fue inhibida en más de un 71,9\% (Figura 2).

Los resultados evidenciaron que en el medio de cultivo Jugo $\mathrm{V} 8$, el comportamiento fue igual por las cepas $\operatorname{Tr} 1 \mathrm{y}$ Tr2 contra las cepas As1, As2 y As3; creciendo cada Trichoderma sp. en toda la superficie del medio y reduciendo la colonia de cada Aspergillus sp. según los enfrentamientos. Esto mismo se observó entre en los enfrentamientos de As 1 y Tr3, pero el efecto antagónico entre As3 y Tr3 fue que el primero colonizó toda la colonia del segun-

Cuadro 4. Antagonismo entre las cepas de Aspergillus sp. y Trichoderma sp.

\begin{tabular}{|c|c|c|c|c|}
\hline \multirow{2}{*}{ Código: cepas } & \multirow{2}{*}{ Hongos } & \multicolumn{3}{c|}{ Clase (Notas 1) } \\
\cline { 3 - 5 } & & PDA & Jugo V8 & Miel-Avena \\
\hline As1 vs. Tr1 & Aspergillus sp./Trichoderma sp. & Clase 4 & Clase 1 & Clase 1 \\
\hline As1 vs. Tr2 & Aspergillus sp./Trichoderma sp. & Clase 1 & Clase 1 & Clase 1 \\
\hline As1 vs. Tr3 & Aspergillus sp./Trichoderma sp. & Clase 4 & Clase 1 & Clase 1 \\
\hline As2 vs. Tr1 & Aspergillus sp./Trichoderma sp. & Clase 2 & Clase 2 & Clase 1 \\
\hline As2 vs. Tr2 & Aspergillus sp./Trichoderma sp. & Clase 2 & Clase 2 & Clase 1 \\
\hline As2 vs. $\operatorname{Tr} 3$ & Aspergillus sp./Trichoderma sp. & Clase 5 & Clase 3 & Clase 3 \\
\hline As3 vs. Tr1 & Aspergillus sp./Trichoderma sp. & Clase 1 & Clase 2 & Clase 1 \\
\hline As3 vs. Tr2 & Aspergillus sp./Trichoderma sp. & Clase 1 & Clase 2 & Clase 1 \\
\hline As3 vs. Tr3 & Aspergillus sp./Trichoderma sp. & Clase 2 & Clase 4 & Clase 2 \\
\hline
\end{tabular}

Cuadro 5. Antagonismo entre los promisorios biocontroladores contra Sclerotinia sp.

\begin{tabular}{|c|c|c|}
\hline Código: cepas & Hongos & Clase (Notas 2) \\
\hline As1 & Aspergillus sp. & Clase 2 \\
\hline Tr1 & Trichoderma sp. & Clase 3 \\
\hline As2 & Aspergillus sp. & Clase 2 \\
\hline Tr2 & Trichoderma sp. & Clase 1 \\
\hline As3 & Aspergillus sp. & Clase 5 \\
\hline Tr3 & Trichoderma sp. & Clase 2 \\
\hline As1+As2+Tr1 & Aspergillus sp., Aspergillus sp. y Trichoderma sp. & Clase 2 \\
\hline As1+As2+Tr2 & Aspergillus sp., Aspergillus sp. y Trichoderma sp. & Clase 1 \\
\hline As1+As2+Tr3 & Aspergillus sp., Aspergillus sp. y Trichoderma sp. & Clase 3 \\
\hline
\end{tabular}


do, creciendo en toda la caja de Petri. En cuanto al enfrentamiento As2 y Tr3, el Aspergillus sp. creció en 2/3 de la caja de Petri y el Trichoderma sp. en el espacio restante sin superponerse las colonias.

En relación con los resultados obtenidos al enfrentar los hongos promisorios como biocontroladores contra Sclerotinia sp. de manera individual y en consorcios (Cuadro 5), la cepa As3 no tuvo un efecto inhibitorio contra el fitopatógeno, por el contrario, Scl generó hasta un $200 \%$ de estructuras de resistencia (Figura 2), por esto no se incluyó en los consorcios. En el enfrentamiento individual las cepas As1, As2 y Tr3 tuvieron un mayor porcentaje de inhibición sobre Sclerotinia sp., seguidos con una menor inhibición por la cepa Tr1; pero fue la cepa Tr2 quien logró inhibir en mayor porcentaje el fitopatógeno (Figura 2).

Figura 2. Control de crecimiento Sclerotinia sp. en medio jugo V8.

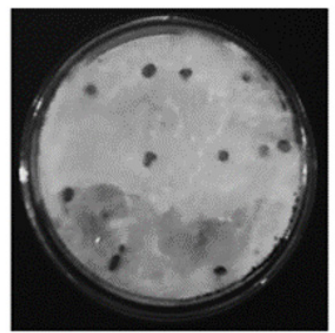

Scl. (control)

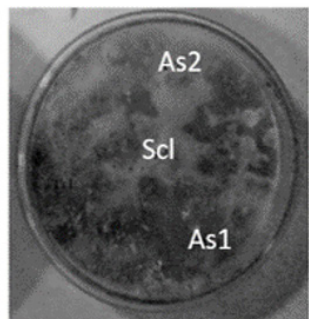

As1+As2+Tr1

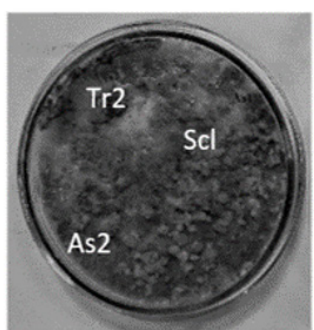

As $1+$ As $2+\operatorname{Tr} 2$

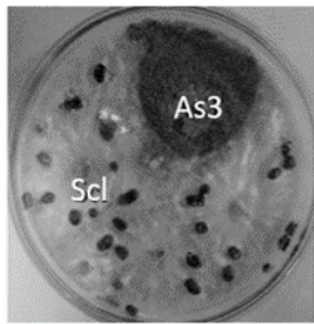

As3 Vs. Scl

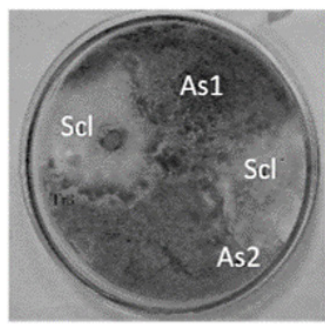

As $1+\mathrm{As} 2+\operatorname{Tr} 3$

Los resultados evidenciaron que el comportamiento de los consorcios frente al fitopatógeno en los tres medios de cultivo fue similar, y se encontró que el consorcio más eficiente para controlar Sclerotinia sp. fue As1+As2+Tr2; en donde se apreció un aumento de la esporulación en el tiempo de la cepa Tr2, seguida por el consorcio As1+As2+Tr1, y el consorcio As1+A2+Tr3. En este último la inhibición de Sclerotinia sp. fue hasta 1/3 de la superficie de los medios de cultivo y se apreció como no se superpusieron las colonias entre sí.

En los antagonismos evaluados enfrentando las cepas promisorias como biocontroladores, en un 66,5\% fueron dominados por las cepas del hongo Trichoderma sp. y en el 23,2\% por las cepas del hongo Aspergillus sp. Según la literatura, esto puede explicarse por la cantidad de enzimas hidrolíticas tales como las lignocelulolíticas, quitinolíticas, proteolíticas, y glucanolíticas producidas por Trichoderma sp., las cuales son excretadas como complejos enzimáticos y están involucradas en procesos como autólisis, germinación de esporas, ramificación y desarrollo de micelios, crecimiento de hifas, nutrición, separación de células y micoparasitismo, procesos esenciales en el control biológico. Igualmente, por la producción de metabolitos secundarios que cumplen funciones de supervivencia para los procesos de competencia, simbiosis, patogenicidad y autoprotección [40,41]. Los hongos del género Aspergillus sp. también se caracterizan por producir enzimas hidrolíticas, la mayoría con gran potencial biotecnológico por su capacidad de degradar paredes celulares de plantas y producir ácidos orgánicos [40].

En los cultivos de los enfrentamientos entre los consorcios y el fitopatógeno no se observó la presencia de esclerocios sin melanina y con melanina, característicos del crecimiento de Sclerotinia sp. para su proceso de reproduc- 
ción asexual después de los 5 a 7 días de cultivo en los que normalmente se forman, de acuerdo con lo reportado por Sousa-Melo et al. [11]. Esto coincidió con los resultados obtenidos, puesto que en el control del fitopatógeno, estas estructuras se formaron a partir del octavo día de incubación. En los tratamientos mencionados no sé formaron esclerocios. La disminución del crecimiento del micelo de Sclerotinia sp. fue mayor empleando a Trichoderma sp. y Aspergillus sp. en consorcios, pero al emplearlos en conjunto se inhibió la formación de esclerocios. Estas estructuras son la principal vía de infección del fitopatógeno y cuando germina puede hacerlo como micelio o cuerpo carpogénico. Asimismo, estas estructuras tienen la capacidad de permanecer viables por largos periodos de tiempo, y son resistentes a cambios adversos físicos y químicos, como a la degradación biológica [42].

La inhibición de Sclerotinia sp. por Trichoderma sp. ha sido ampliamente estudiado [33, 40-42], pero no ocurre lo mismo con Aspergillus sp. donde son pocos los estudios que evalúan el hongo Aspergillus sp. como antagonista de fitopatógenos [36, 37]. En este estudio, se pudo evidenciar con los consorcios de los hongos Trichoderma sp. y Aspergillus sp. frente a Sclerotinia sp., que la eficiencia de inhibición del fitopatógeno causante de la podredumbre blanca es más eficaz al trabajar con metabolismos provenientes de estos dos tipos de hongos. Esto coincide con lo descrito por Cologna et al. [40], respecto al uso combinado de diversas enzimas provenientes de cuatro especies de Aspergillus sp. con Trichoderma reesei para establecer el grado de sacarificación de la cascara de trigo, reportando que fue mayor el grado de sacarificación empleando el coctel de enzimas de Aspergillus sp. y Trichoderma sp., que cuando la cascara de trigo fue tratada solo con el hongo T. reesei. Estos resultados apoyan la conjetura sobre la eficiencia metabólica al emplear combinaciones de enzimas mixtas (i.e., enzimas del metabolismo primario y metabolitos secundarios).

\section{Análisis estadístico}

El análisis del conglomerado jerárquico mediante la medida de similaridad de Jaccard permitió establecer la semejanza entre las relaciones antagónicas y su efecto sobre el fitopatógeno. Se obtuvo que en el consorcio As1+As2+Tr1 fueron mutualistas las cepas en un 95,1\%, el consorcio solo logró inhibir en un 66,5\% el crecimiento de Sclerotinia sp. Individualmente, las cepas As1 y Tr1 inhibieron en un 60,6\% y en un 91,0\% a As2 y $\operatorname{Tr} 1$, respectivamente; datos que fueron confirmados al realizar un análisis de varianza de un factor y la prueba Tukey, en donde se pudo encontrar que hubo una asociación entre estas dos cepas de hasta el 60,6\% (en promedio), de acuerdo con la semejanza intergrupal realizada con un nivel de confianza del 95\%.

Estadísticamente, el comportamiento del consorcio As1+As2+Tr2 fue similar entre las cepas con una eficiencia de inhibición de Scl. del 85,1\% en conjunto, y de una inhibición del 95,6\% y 95,11\% por las cepas As1 y Tr2, respectivamente. Sin embargo, el grado de afinidad por estas dos cepas no fue superior al $9,66 \%$, por lo cual en el tratamiento As1+As2+Tr2 contra Scl. disminuyó hasta un 12,9\% el patógeno, de acuerdo con la comparación intergrupal de Tukey (Cuadro 6).

Se evidenció que en el consorcio As1+As2+Tr3, la inhibición no fue superior al 50,0\%, aunque las cepas de Aspergillus sp. fueron mutualistas entre ellas en un 85,7\%. Sin embargo, la cepa Tr3 tuvo un crecimiento inferior al $28,1 \%$ y el efecto inhibidor sobre el fitopatógeno individualmente fue superior al 50,0\% para cada cepa. Esto se evidenció en la comparación de medias porcentuales referente a la inhibición del fitopatógeno mediante un análisis de varianza y comparación intergrupal con prueba Tukey (Cuadro 7).

Con la prueba estadística de Tukey se corroboró que hubo un porcentaje de asociación del 66,5\% entre las cepas As1, As2 y Tr1, y de 87,14\% entre las cepas As1 As2 y Tr2; ambos consorcios con un nivel de confianza del 95\% (Cuadro 7). Para todos los datos, se realizó una comprobación de homogeneidad, encontrando la correcta distribución de estos y sin variaciones atípicas al estudio realizado.

De acuerdo con el análisis de varianza y la prueba de Tukey, los tratamientos en consorcio fueron semejantes, aportando un grado de inhibición entre el $47,3-85,1 \%$, en dónde se pudo encontrar que el cambio de la cepa de Trichoderma sp. aportó en mayor o menor grado al control del fitopatógeno, lo cual se observó en los tratamientos individuales contra Sclerotinia sp., ya que los hongos As1 y Tr2 fueron mutualistas hasta en un 9,66\%, razón 
por la cual el tratamiento del consorcio As1+As2+Tr2 solo fue efectivo hasta el $85 \%$. También para As1 y As2, y As2 y Tr2 hubo un grado de afinidad de hasta el 70,9\%, en ambas combinaciones.

Cuadro 6. Prueba Tukey enfrentamientos individuales contra Sclerotinia sp.

\begin{tabular}{|c|c|c|c|c|c|c|}
\hline \multirow{2}{*}{ (I) Trat. } & \multirow{2}{*}{ (J) Trat. } & \multirow{2}{*}{$\begin{array}{c}\text { Diferencia/medias } \\
\text { (I-J) }\end{array}$} & \multirow{2}{*}{ Desv. Error } & \multirow{2}{*}{ Sig. } & \multicolumn{2}{|c|}{ IC al 95\% } \\
\hline & & & & & L. Inf. & L. Sup. \\
\hline \multirow{6}{*}{ As1 } & $\operatorname{Tr} 1$ & $41,3 \%^{*}$ & $0,59 \%$ & $7,62 \mathrm{E}-13$ & $39,4 \%$ & $43,2 \%$ \\
\hline & As2 & $30,4 \%^{*}$ & $0,59 \%$ & $7,62 \mathrm{E}-13$ & $28,5 \%$ & $32,3 \%$ \\
\hline & $\operatorname{Tr} 2$ & $-0,44 \%$ & $0,59 \%$ & 0,99 & $-2,32 \%$ & $1,44 \%$ \\
\hline & As3 & $83,7 \%^{*}$ & $0,59 \%$ & $7,62 \mathrm{E}-13$ & $81,8 \%$ & $85,6 \%$ \\
\hline & $\operatorname{Tr} 3$ & $25,1 \%^{*}$ & $0,59 \%$ & $7,62 \mathrm{E}-13$ & $23,2 \%$ & $26,9 \%$ \\
\hline & $\mathrm{Scl}$ & $95,1 \%^{*}$ & $0,59 \%$ & $7,62 \mathrm{E}-13$ & $93,2 \%$ & $96,9 \%$ \\
\hline \multirow{6}{*}{$\operatorname{Tr} 1$} & As1 & $-41,3 \%^{*}$ & $0,59 \%$ & $7,62 \mathrm{E}-13$ & $-43,2 \%$ & $-39,4 \%$ \\
\hline & As2 & $-10,8 \%{ }^{*}$ & $0,59 \%$ & $7,62 \mathrm{E}-13$ & $-12,7 \%$ & $-9,01 \%$ \\
\hline & $\operatorname{Tr} 2$ & $-41,7 \% *$ & $0,59 \%$ & $7,62 \mathrm{E}-13$ & $-43,6 \%$ & $-39,8 \%$ \\
\hline & As3 & $42,4 \%^{*}$ & $0,59 \%$ & $7,62 \mathrm{E}-13$ & $40,5 \%$ & $44,3 \%$ \\
\hline & $\operatorname{Tr} 3$ & $-16,2 \%^{*}$ & $0,59 \%$ & $7,62 \mathrm{E}-13$ & $-18,1 \%$ & $-14,3 \%$ \\
\hline & $\mathrm{Scl}$ & $53,7 \%{ }^{*}$ & $0,59 \%$ & $7,62 \mathrm{E}-13$ & $51,8 \%$ & $55,6 \%$ \\
\hline \multirow{6}{*}{ As2 } & As1 & $-30,4 \%^{*}$ & $0,59 \%$ & $7,62 \mathrm{E}-13$ & $-32,3 \%$ & $-28,5 \%$ \\
\hline & $\operatorname{Tr} 1$ & $10,8 \%^{*}$ & $0,59 \%$ & $7,62 \mathrm{E}-13$ & $9,0 \%$ & $12,7 \%$ \\
\hline & Tr2 & $-30,8 \%$ & $0,59 \%$ & $7,62 \mathrm{E}-13$ & $-32,7 \%$ & $-29,0 \%$ \\
\hline & As3 & $53,3 \%^{*}$ & $0,59 \%$ & $7,62 \mathrm{E}-13$ & $51,4 \%$ & $55,2 \%$ \\
\hline & Tr3 & $-5,33 \%{ }^{*}$ & $0,59 \%$ & $1,95 \mathrm{E}-8$ & $-7,2 \%$ & $-3,4 \%$ \\
\hline & $\mathrm{Scl}$ & $64,6 \%{ }^{*}$ & $0,59 \%$ & $7,62 \mathrm{E}-13$ & $62,7 \%$ & $66,5 \%$ \\
\hline \multirow{6}{*}{ Tr2 } & As1 & $0,44 \%$ & $0,59 \%$ & 0,99 & $-1,4 \%$ & $2,32 \%$ \\
\hline & $\operatorname{Tr} 1$ & $41,7 \%^{*}$ & $0,59 \%$ & $7,62 \mathrm{E}-13$ & $39,8 \%$ & $43,6 \%$ \\
\hline & As2 & $30,8 \%{ }^{*}$ & $0,59 \%$ & $7,62 \mathrm{E}-13$ & $29,0 \%$ & $32,7 \%$ \\
\hline & As3 & $84,2 \%{ }^{*}$ & $0,59 \%$ & $7,62 \mathrm{E}-13$ & $82,3 \%$ & $86,1 \%$ \\
\hline & Tr3 & $25,5 \%^{*}$ & $0,59 \%$ & $7,62 \mathrm{E}-13$ & $23,6 \%$ & $27,4 \%$ \\
\hline & $\mathrm{Scl}$ & $95,5 \%^{*}$ & $0,59 \%$ & $7,62 \mathrm{E}-13$ & $93,6 \%$ & $97,4 \%$ \\
\hline \multirow{6}{*}{ As3 } & As1 & $-83,7 \%{ }^{*}$ & $0,59 \%$ & $7,62 \mathrm{E}-13$ & $-85,6 \%$ & $-81,8 \%$ \\
\hline & $\operatorname{Tr} 1$ & $-42,4 \%{ }^{*}$ & $0,59 \%$ & $7,62 \mathrm{E}-13$ & $-44,3 \%$ & $-40,5 \%$ \\
\hline & As2 & $-53,3 \% *$ & $0,59 \%$ & $7,62 \mathrm{E}-13$ & $-55,2 \%$ & $-51,4 \%$ \\
\hline & Tr2 & $-84,2 \%{ }^{*}$ & $0,59 \%$ & $7,62 \mathrm{E}-13$ & $-86,1 \%$ & $-82,3 \%$ \\
\hline & Tr3 & $-58,6 \%^{*}$ & $0,59 \%$ & $7,62 \mathrm{E}-13$ & $-60,5 \%$ & $-56,7 \%$ \\
\hline & $\mathrm{Scl}$ & $11,3 \%^{*}$ & $0,59 \%$ & $7,62 \mathrm{E}-13$ & $9,44 \%$ & $13,2 \%$ \\
\hline \multirow{6}{*}{$\operatorname{Tr} 3$} & As1 & $-25,1 \%$ & $0,59 \%$ & $7,62 \mathrm{E}-13$ & $-26,9 \%$ & $-23,2 \%$ \\
\hline & $\operatorname{Tr} 1$ & $16,2 \%^{*}$ & $0,59 \%$ & $7,62 \mathrm{E}-13$ & $14,3 \%$ & $18,1 \%$ \\
\hline & As2 & $5,33 \%^{*}$ & $0,59 \%$ & $1,95 \mathrm{E}-8$ & $3,44 \%$ & $7,21 \%$ \\
\hline & Tr2 & $-25,5 \%$ & $0,59 \%$ & $7,62 \mathrm{E}-13$ & $-27,4 \%$ & $-23,6 \%$ \\
\hline & As3 & $58,6 \%^{*}$ & $0,59 \%$ & $7,62 \mathrm{E}-13$ & $56,7 \%$ & $60,5 \%$ \\
\hline & $\mathrm{Scl}$ & $70,0 \%{ }^{*}$ & $0,59 \%$ & $7,62 \mathrm{E}-13$ & $68,1 \%$ & $71,8 \%$ \\
\hline \multirow{6}{*}{ Scl } & As1 & $-95,1 \%{ }^{*}$ & $0,59 \%$ & $7,62 \mathrm{E}-13$ & $-96,9 \%$ & $-93,2 \%$ \\
\hline & Tr1 & $-53,7 \%$ & $0,59 \%$ & $7,62 \mathrm{E}-13$ & $-55,6 \%$ & $-51,8 \%$ \\
\hline & As2 & $-64,6 \%$ & $0,59 \%$ & $7,62 \mathrm{E}-13$ & $-66,5 \%$ & $-62,7 \%$ \\
\hline & $\operatorname{Tr} 2$ & $-95,5 \%{ }^{*}$ & $0,59 \%$ & $7,62 \mathrm{E}-13$ & $-97,4 \%$ & $-93,6 \%$ \\
\hline & As3 & $-11,3 \%{ }^{*}$ & $0,59 \%$ & $7,62 \mathrm{E}-13$ & $-13,2 \%$ & $-9,44 \%$ \\
\hline & Tr3 & $-70,0 \%{ }^{*}$ & $0,59 \%$ & $7,62 \mathrm{E}-13$ & $-71,8 \%$ & $-68,1 \%$ \\
\hline
\end{tabular}


Cuadro 7. Prueba Tukey consorcios contra Sclerotinia sp.

\begin{tabular}{|c|c|c|c|c|c|c|}
\hline \multirow{2}{*}{ (I) \# Trat. } & \multirow{2}{*}{ (J) \# Trat. } & \multirow{2}{*}{$\begin{array}{c}\text { Diferencia de } \\
\text { medias (I-J) }\end{array}$} & \multirow{2}{*}{ Desv. Error } & \multirow{2}{*}{ Sig. } & \multicolumn{2}{|c|}{ Intervalo de confianza al $95 \%$} \\
\hline & & & & & Lím. Inf. & Lím. Sup. \\
\hline \multirow{3}{*}{$\mathrm{Scl}$} & As1+As2+Tr1 & $-64,4 \%{ }^{*}$ & $0,71 \%$ & $1,08 \mathrm{E}-12$ & $-66,4 \%$ & $-62,4 \%$ \\
\hline & As1+As2+Tr2 & $-85,1 \%{ }^{*}$ & $0,71 \%$ & $1,08 \mathrm{E}-12$ & $-87,1 \%$ & $-83,1 \%$ \\
\hline & As1+As2+Tr3 & $-47,3 \% *$ & $0,71 \%$ & $1,08 \mathrm{E}-12$ & $-49,3 \%$ & $-45,2 \%$ \\
\hline \multirow{3}{*}{ As1+As2+Tr1 } & $\mathrm{Scl}$ & $64,4 \%^{*}$ & $0,71 \%$ & $1,08 \mathrm{E}-12$ & $62,4 \%$ & $66,4 \%$ \\
\hline & As1+As2+Tr2 & $-20,6 \%^{*}$ & $0,71 \%$ & $1,09 \mathrm{E}-12$ & $-22,7 \%$ & $-18,6 \%$ \\
\hline & As1+As2+Tr3 & $17,1 \%^{*}$ & $0,71 \%$ & $1,34 \mathrm{E}-12$ & $15,1 \%$ & $19,1 \%$ \\
\hline \multirow{3}{*}{ As1+As2+Tr2 } & $\mathrm{Scl}$ & $85,1 \% *$ & $0,71 \%$ & $1,08 \mathrm{E}-12$ & $83,1 \%$ & $87,1 \%$ \\
\hline & As1+As2+Tr1 & $20,6 \%^{*}$ & $0,71 \%$ & $1,09 \mathrm{E}-12$ & $18,6 \%$ & $22,7 \%$ \\
\hline & As1+As2+Tr3 & $37,7 \%^{*}$ & $0,71 \%$ & $1,08 \mathrm{E}-12$ & $35,7 \%$ & $39,8 \%$ \\
\hline \multirow{3}{*}{ As $1+$ As2+Tr3 } & $\mathrm{Scl}$ & $47,3 \%^{*}$ & $0,71 \%$ & $1,08 \mathrm{E}-12$ & $45,2 \%$ & $49,3 \%$ \\
\hline & As1+As2+Tr1 & $-17,1 \%{ }^{*}$ & $0,71 \%$ & $1,34 \mathrm{E}-12$ & $-19,1 \%$ & $-15,1 \%$ \\
\hline & As1+As2+Tr2 & $-37,7 \%{ }^{*}$ & $0,71 \%$ & $1,08 \mathrm{E}-12$ & $-39,8 \%$ & $-35,7 \%$ \\
\hline
\end{tabular}

Por otro lado, las cepas As2 y $\operatorname{Tr} 1$ fueron mutualistas hasta en un 90,9\%, sin embargo, As1 y $\operatorname{Tr} 1$ se inhibieron entre sí en un 60,6\%, por lo que el consorcio As1+As2+Tr1 solo logró un grado de efectividad de hasta el 66,5\%. Por último, Tr3 fue mutualista con As1 y As2 en 76,8\% y 7,21\%, respectivamente, por esta razón, el grado de control de este consorcio sobre el fitopatógeno disminuyó considerablemente hasta el 47,1\%. No obstante, cabe resaltar que en este último tratamiento tampoco se formaron estructuras de resistencia (esclerocios), pero si un mayor crecimiento del micelio de Scl.

\section{CONCLUSIONES}

Los resultados sugieren que la mejor combinación de las cepas de Aspergillus sp. y Trichoderma sp. para controlar el crecimiento de Sclerotinia sp. in vitro, aislado de lechugas enfermas por este fitopatógeno, es el consorcio As1+As2+Tr2 (eficiencia de inhibición = 85,1\%), clasificación clase 1 en la escala de notas 2 modificada. Adicionalmente, en el enfrentamiento individual las mejores cepas con efecto antagónico contra el fitopatógeno Sclerotinia sp., posiblemente son las cepas Tr2 y As 1 de los géneros Trichoderma sp. y Aspergillus sp. con una inhibición del 95,6\% y 95,1\%, respectivamente, cada una de acuerdo con el análisis de similaridad de Jaccard. La inhibición de los esclerocios de Sclerotinia sp. por los consorcios de Trichoderma sp. y Aspergillus sp. es probablemente una reducción de la patogenicidad del hongo al romper el ciclo infeccioso.

Finalmente, es relevante continuar estudiando los mecanismos de antagonismo de las cepas As1, As2 y Tr2 sobre Sclerotinia sp., la asociación metabólica del consorcio y la evaluación in vivo del consorcio como control biológico de Sclerotinia sp. en cultivos de lechuga (Lactuca sativa) para establecer su viabilidad y ecotoxicidad al pensar en un producto biológico que pueda ser empleado en el control integrado del manejo de plagas de cultivos de lechuga.

\section{AGRADECIMIENTOS}

Los autores agradecen al Centro de Investigaciones y Desarrollo Científico (CIDC) de la Universidad Distrital Francisco José de Caldas por la financiación del proyecto código: 3-180-241-11. 


\section{REFERENCIAS}

[1] JIMENEZ-SANTOS, JOSÉ-ESTALIN. Aplicación de cuatro dosis de fertilizante (micromate calcium fortified) a base de micro elementos en el cultivo de lechuga (Lactuca sativa L.) variedad great lakes 659, bajo condiciones agroecológicas en la provincia de Lamas [Bachelor Thesis]. Tarapoto (Perú): Universidad Nacional de San Martín, Facultad de Ciencias Agrarias, 2016, 71 p.

[2] KIM, MOO-JUNG; MOON, YOUYOUN; TOU, JANET; MOU, BEIQUAN; WATERLAND, NICOLE. Nutritional value, bioactive compounds and health benefits of lettuce (Lactuca sativa L.). Journal of Food Composition and Analysis, v. 49, 2016, p. 19-34. https://doi.org/10.1016/j.jfca.2016.03.004

[3] PARISMORENO-RIVAS, LAURA. Evaluación de tres niveles de nitrato de potasio en lechuga Lactuca sativa L. cultivado bajo sistema de producción hidropónica [Bachelor Thesis]. Guayaquil (Ecuador): Facultad de Ciencias Agrarias, Universidad de Guayaquil, 2017, 77 p.

[4] ASSEFA, AWRARIS-DERBIE; CHOI, SUSANNA; LEE, JAE-EUN; SUNG, JUNG-SOOK; HUR, ON-SOOK; RO, NA-YOUNG; LEE, HO-SUNG; JANG, SUK-WOO; RHEE, JU-HEE. Identification and quantification of selected metabolites in differently pigmented leaves of lettuce (Lactuca sativa L.) cultivars harvested at mature and bolting stages. BMC Chemistry, v. 13, n. 56, 2019, p. 1-15. https://doi.org/10.1186/s13065-019-0570-2.

[5] COLOMBIA. AGRONET-MINISTERIO DE AGRICULTURA Y DESARROLLO RURAL. Reporte: área. Producción, rendimiento y participación municipal en el departamento por cultivo [online]. 2020. https://www.agronet.gov.co/estadistica/Paginas/home.aspx?cod=4 [citado 29 de septiembre de 2020].

[6] COLOMBIA. AGRONET-MINISTERIO DE AGRICULTURA Y DESARROLLO RURAL. Reporte: área, producción y rendimiento nacional por cultivo [online]. 2020. https://www.agronet.gov.co/estadistica/Paginas/home.aspx?cod=1 [citado 29 de septiembre de 2020].

[7] PÉREZ, SILVIA-LILIAM; PIEDRAHÍTA, WILSON; ARBELÁEZ, GERMÁN. Patogénesis de la pudrición blanda de la lechuga (Lactuca sativa L.) en la Sabana de Bogotá causada por Sclerotinia sclerotiorum (Lib.) de Bary y Sclerotinia minor Jagger. Una revisión. Revista Colombiana de Ciencias Hortícolas, v. 3, n. 2, 2009, p. 262-274. https://doi.org/10.17584/rcch.2009v3i2.1217

[8] AZEVEDO, LUCIANA; CHAGAS-PAULA, DANIELA-APARECIDA; KIM, HYEMEE; MONTEIRO-ROQUE, ALINA-CRISTINA; TRANCHES-DIAS, KRIS-SIMONE; CRUZ-MACHADO, JOSÉ; GOMES-SOARES, MARISI; MERTENS-TALCOTT, SUSANNE. White mold (Sclerotinia sclerotiorum), friend or foe: Cytotoxic and mutagenic activities in vitro and in vivo. Food Research International, v. 80, 2016, p. 27-35. https://doi.org/10.1016/j.foodres.2015.11.029

[9] SUN, GUANGZHENG; YAO, TUO; FENG, CHANJING; CHEN, LONG; LI, JIANHONG; WANG, LIDE. Identification and biocontrol potential of antagonistic bacteria strains against Sclerotinia sclerotiorum and their growth-promoting effects on Brassica napus. Biological control, v. 104, 2017, p. 35-43. https://doi.org/10.1016/j.biocontrol.2016.10.008

[10] DELGADO-ORTIZ, JUAN-CARLOS; OCHOA-FUENTES, YISA-MARÍA; BELTRÁN-BEACHE, MARIANA; CERNA-CHÁVEZ, ERNESTO. Evaluación in vitro de la actividad antifungica del extracto de Heliopsis longipes en cepas de Sclerotium cepivorum y Sclerotinia sclerotiorum. Revista mexicana de ciencias agrícolas, v. 9, n. 2, 2018, p. 871-877. http://dx.doi.org/10.29312/remexca.v9i4.337

[11] SOUSA-MELO, BRUNA; VOLTAN, ALINE-RAQUEL; ARRUDA, WALQUIRIA; CARDOSO-LOPES, FABYANO-ALVARES; CASTRO-GEORG, RAPHAELA; ULHOA, CIRANO-JOSÉ. Morphological and molecular aspects of sclerotial development in the phytopathogenic fungus Sclerotinia sclerotiorum. Microbiological Research, v. 229, 2019, p. 126326.

https://doi.org/10.1016/j.micres.2019.126326Get

[12] ARGÜELLO-ARIAS, HELIODORO. Producción limpia de lechuga: cartilla para productores. 1 ed. Bogotá (Colombia): Universidad Nacional de Colombia, 2011, 23 p.

[13] BERNARDINO-HERNÁNDEZ, HÉCTOR-ULISES; MARIACA-MÉNDEZ, RAMÓN; NAZAR-BEUTELSPACHER, AUSTREBERTA; ÁLVAREZ-SOLÍS, JOSÉ-DAVID; TORRES-DOSAL, ARTURO; HERRERA-PORTU- 
GAL, CRISPÍN. Conocimientos, conductas y síntomas de intoxicación aguda por plaguicidas entre productores de tres sistemas de producción agrícolas en los altos de Chiapas, México. Revista Internacional de Contaminación Ambiental, v. 35, n. 1, 2019, p. 7-23.

http://dx.doi.org/10.20937/RICA.2019.35.01.01

[14] STENBERG, JOHAN. A Conceptual Framework for Integrated Pest Management. Trends in Plant Science, v. 22, n. 9, 2017, p. 759-769. http://dx.doi.org/10.1016/j.tplants.2017.06.010

[15] DE SILVA, NIMALI; BROOKS, SIRAPRAPA; LUMYONG, SAISAMORN; HYDE, KEVIN. Use of endophytes as biocontrol agents. Fungal Biology Reviews, v. 33, n. 2, 2019, p. 133-148. https://doi.org/10.1016/j.fbr.2018.10.001

[16] PINTO-DA SILVA, GERARDA-BEATRIZ; HECKLER, LEISE-INES; DURIGON, MIRIA; DOS SANTOS, RICARDO-FELICIANO; LOVATO, MAIKE; FINGER, GEÍSA; BLUME, ELENA. Biological control of white mold ('Sclerotinia sclerotiorum') in lettuce using Brazilian 'Trichoderma' spp. Strains. Australian Journal of Crop Science, v. 13, n. 6, 2019, p. 803-809.

[17] SEGARAN, GAYATHRI; SATHIAVELU, MYTHILI. Fungal endophytes: A potent biocontrol agent and a bioactive metabolites reservoir. Biocatalysis and Agricultural Biotechnology, v. 21, 2019, p. 1-17. https://doi.org/10.1016/j.bcab.2019.101284

[18] YANG, FAN; ABDELNABBY, HAZEM; XIAO, YANNONG. A mutant of the nematophagous fungus Paecilomyces lilacinus [3] is a novel biocontrol agent for Sclerotinia sclerotiorum. Microbial pathogenesis, v. 89, 2015, p. 169-176. https://doi.org/10.1016/j.micpath.2015.10.012

[19] ELSHESHTAWI, MOHAMED; ELKHAKY, MAGED; SAYED, SHABAN; BAHKALI, ALI; MOHAMMED, ARIF; GAMBHIR, DIKSHIT; MANSOUR, AREF; ELGORBAN, ABDALLAH. M. Integrated control of white rot disease on beans caused by Sclerotinia sclerotiorum using Contans ${ }^{\circledR}$ and reduced fungicides application. Saudi journal of biological sciences, v. 24, n. 2, 2017, p. 405-409. https://doi.org/10.1016/j.sjbs.2016.01.038

[20] SABATÉ, DANIELA; PÉREZ-BRANDAN, CAROLINA; PETROSELLI, GABRIELA; ERRA-BALSELLS, ROSA; AUDISIO, CARINA. Biocontrol of Sclerotinia sclerotiorum (Lib.) de Bary on common bean by native lipopeptide-producer Bacillus strains. Microbiological research, v. 211, 2018, p. 21-30. https://doi.org/10.1016/j.micres.2018.04.003

[21] SUN, ZHAN-BIN; WANG, QI; ZHANG, UN; JIANG, WEI-ZHI; LI, SHI-DONG; MA, GUI-ZHEN; SUN, MANHONG. The transcription factor-encoding gene $\mathrm{crtf}$ is involved in Clonostachys chloroleuca mycoparasitism on Sclerotinia sclerotiorum. Microbiological research, v. 210, 2018, p. 6-11. https://doi.org/10.1016/j.micres.2018.03.002

[22] CLINE, MARLIN. Principles of soil sampling. Soil Science, v. 58, n. 2, 1944, p. 275-288.

[23] JAKLITSCH, WALTER. European species of Hypocrea Part I. The green-spored species". Studies in mycology, v. 63, 2009, p. 1-91. 10.3114/sim.2009.63.01

[24] JAKLITSCH, WALTER. European species of Hypocrea part II: species with hyaline ascospores. Fungal diversity, v. 48, n. 1, 2011, p. 1-250. 10.1007/s13225-011-0088-y

[25] ZHU, Z.X.; ZHUANG, W.Y. Trichoderma (Hypocrea) species with green ascospores from China. Persoonia, v. 34, 2015, p. 113-129. $10.3767 / 003158515 \times 686732$

[26] SAMSON, R.A.; VISAGIE, C.M.; HOUBRAKEN, J.; HONG, S.B.; HUBKA, V.; KLAASSEN, C.H.W.; PERRONE, G.; SEIFERT, K.A.; SUSCA, A.; TANNEY, J.B.; VARGA, J.; KOCSUBÉ, S.; SZIGETI, G.; YAGUCHI, T.; FRISVAD, J.C. Phylogeny, identification and nomenclature of the genus Aspergillus. Studies in mycology, v. 78, 2014, p. 141-173. 10.1016/j.simyco.2014.07.004

[27] SKLENÁŘ, F.; JURJEVIC, Z.; ZALAR, P.; FRISVAD, J.C.; VISAGIE, C.M.; KOLARIK, M.; HOUBRAKEN, J.; CHEN, A.J.; YILMAZ, N.; SEIFERT, K.A.; COTON, M.; DÉNIEL, F.; GUNDE-CIMERMAN, N.; SAMSON, R.A.; 
PETERSON, S.W.; HUBKA, V. Phylogeny of xerophilic aspergilli (subgenus Aspergillus) and taxonomic revision of section Restricti. Studies in mycology, v. 88, 2017, p. 161-236.

10.1016/j.simyco.2017.09.002

[28] MORENO, CARLOS-ANDRÉS; COTES-PRADO, ALBA-MARINA. Desarrollo de un bioplaguicida a base de Trichoderma koningiopsis Th003 y uso en el cultivo de la lechuga para el control del moho blanco (Sclerotinia sclerotiorum y Sclerotinia minor). $1^{\text {a }}$ ed. Bogotá (Colombia): CORPOICA, 2010, 80 p.

[29] SÁNCHEZ-COLÍN, M.J. Microbiología de suelos: Técnicas, métodos y medios de cultivo. $1^{\text {a }}$ ed. México D.F. (México): Universidad Nacional Autónoma de México, 2004, 53 p.

[30] SAHARAN, G.S.; MEHTA, NARESH. Sclerotinia diseases of crop plants: Biology, Ecology and Disease Management. Springer Science \& Business Media B.V., 2008, 531 p.

[31] VAILLANT-FLORES, DAYMARA; ALMÁNDOZ-PARRADO, JULIA; GATO-CÁRDENAS, YOANA; RAMÍREZ-OCHOA, REBECA; DÍAZ-RODRÍGUEZ, JOSÉ-ANTONIO. Establecimiento de Trichoderma spp. en sustratos orgánicos empleados en casas de postura de Tecnología de Cultivo Protegido. Fitosanidad, v. 20, n. 2, 2016, p. 95-96.

[32] ROYSE, D.J.; RIES, S.M. The influence of fungi isolated from peach twigs on the pathogenicity of Cytospora cincta. Phytopathology, v. 6882, 1978, p. 603-607.

[33] GUIGÓN-LÓPEZ, CÉSAR; GUERRERO-PRIETO, VÍCTOR; VARGAS-ALBORES, FRANCISCO; CARVAJAL-MILLÁN, ELIZABETH; ÁVILA-QUEZADA, GRACIELA-DOLORES; BRAVO-LUNA, LETICIA; RUOCCO, MICHELINA; LANZUISE, STEFANIA; WOO, SHERIDAN; LORITO, MATTEO. Identificación molecular de cepas nativas de Trichoderma spp. su tasa de crecimiento in vitro y antagonismo contra hongos fitopatógenos. Revista mexicana de fitopatología, v. 28, n. 2, 2010, p. 87-96.

[34] MELLO, SUELI-CORRÊA, ÁVILA, ZILA; LEONARDO-MINARE, BRAÚNA; PÁDUA, RAQUEL; GOMES, DIOGO. Cepas de Trichoderma spp. para el control biológico de Sclerotium rolfsii Sacc. Fitosanidad, v. 11, n. 1,2007, p. 3-9.

[35] VEGA-DIENSTMAIER, JOHANN-MARTÍN; ARÉVALO-FLORES, MARTÍN. Clasificación mediante análisis de conglomerados: un método relevante para la psiquiatría. Revista de Neuro-Psiquiatría, v. 77, n. 1, 2014, p. 31-39.

[36] EL-SAYED, ASHRAF; SHAD-ALI, GUL. Aspergillus flavipes is a novel efficient biocontrol agent of Phytophthora parasitica. Biological control, v. 140, 2020, p. 1-11. https://doi.org/10.1016/j.biocontrol.2019.104072

[37] EL-DEBAIKY, SAMAH. Antagonistic studies and hyphal interactions of the new antagonist Aspergillus piperis against some phytopathogenic fungi in vitro in comparison with Trichoderma harzianum. Microbial pathogenesis, v. 113, 2017, p. 135-143. https://doi.org/10.1016/j.micpath.2017.10.041

[38] PARK, HEE-SOO; JUN, SANG-CHEOL, HAN, KAP-HOON; HONG, SEUNG-BEOM; YU, JAE-HYUK. Diversity, application, and synthetic biology of industrially important Aspergillus fungi. Advances in applied microbiology, v. 100, 2017, p. 161-202.

https://doi.org/10.1016/bs.aambs.2017.03.001

[39] KRIJGSHELD, P.; BLEICHRODT, R.V.; VAN VELUW, G.J.; WANG, F.; MÜLLER, W.H.; DIJKSTERHUIS, J.; WÖSTEN, H.A.B. Development in aspergillus. Studies in mycology, v. 74, 2013, p. 1-29.

https://doi.org/10.3114/sim0006

[40] DI COLOGNA, NICHOLÁS-DE MOJANA; GÓMEZ-MENDOZA, DIANA-PAOLA; FONSECA-ZANOELO, FABIANA; GIANNESI, GIOVANA-CRISTINA; DE ALENCAR-GUIMARAES, NELCIELE-CAVALIERI; DE SOUZA-MOREIRA, LEONORA-RIOS; FERREIRA-FILHO, EDIVALDO-XIMENES; ORNELAS-RICART, CARLOS-ANDRÉ. Exploring Trichoderma and Aspergillus secretomes: proteomics approaches for the identification of enzymes of biotechnological interest. Enzyme and microbial technology, v. 109, 2018, p. 1-10. https://doi.org/10.1016/j.enzmictec.2017.08.007

[41] ZEILINGER, SUSANNE; GRUBER, SABINE; BANSAL, RAVINDRA; MUKHERJEE, PRASUN. Secondary metabolism in Trichoderma-Chemistry meets genomics. Fungal biology reviews, v. 30, n. 2, 2016, p. 74-90. https://doi.org/10.1016/j.fbr.2016.05.001

[42] SMOLIŃSKA, URSZULA; KOWALSKA, BEATA. Biological control of the soil-borne fungal pathogen Sclerotinia sclerotiorum -- a review. Journal of Plant Phatology, v. 100, 2018, p. 1-12.

https://doi.org/10.1007/s42161-018-0023-0 